\title{
Florística e Fitossociologia de uma Floresta Estacional Semidecidual, Reserva Privada Osununú-Misiones, Argentina
}

\author{
Santiago José Elías Velazcoํㅜ, Franklin Galvão ${ }^{1}$, Héctor Alejandro Keller², \\ Natalia Alejandra Bedrij ${ }^{1}$ \\ ${ }^{1}$ Departamento de Ciências Florestais, Universidade Federal do Paraná - UFPR, Curitiba/PR, Brasil \\ ${ }^{2}$ Facultad de Ciencias Forestales, Universidad Nacional de Misiones - UNaM, Eldorado/Mnes, Argentina
}

\begin{abstract}
RESUMO
O estudo foi realizado em um fragmento de Floresta Estacional Semidecidual (Misiones, Argentina), com o objetivo de analisar as variáveis fitossociológicas das espécies arbóreas, por meio da estratificação da área de acordo com a geomorfologia. Inicialmente, baseando-se na declividade e por meio de fotointerpretação, a área foi compartimentalizada em: planície, encostas com declividade média, encostas com declividade alta e topo. Dentro de cada compartimento, foram alocados três conglomerados compostos de quatro subunidades de $100 \mathrm{~m}^{2}$, totalizando 48 subunidades, nas quais foram incluídas todas as árvores com circunferência à altura do peito $\geq 10 \mathrm{~cm}$, sendo também medidas a profundidade do solo e a declividade. Foram amostradas 72 espécies, 59 gêneros e 31 famílias. Foi possível detectar variações das espécies e dos valores de importância, obtidos de acordo o gradiente topográfico. Na Análise de Correspondência Canônica, os valores de cobertura das espécies mostraram ter uma correlação significativa com os parâmetros profundidade de solo e declividade.
\end{abstract}

Palavras-chave: fitossociologia, estrato arbóreo, gradiente topográfico.

\section{Floristic and Phytosociology in a Semideciduous Seasonal Forest - Osununú Private Reserve, Misiones, Argentina}

\begin{abstract}
This survey was conducted in a fragment of Semideciduous Seasonal Forest (Misiones, Argentina) in order to analyze the phytosociological variable of tree species by means of area stratification according to geomorphology. Initially, based on the slope and through photointerpretation, the area was compartmentalized in lowland, hillsides with intermediate slopes and high slopes, and highlands. Within each compartment, three plots composed of four $100 \mathrm{~m}^{2}$ sub-units were allocated; totaling 48 sub-units, where all trees with circumference at breast height $\geq 10 \mathrm{~cm}$ were included, and soil depth and slope were measured. Sampling comprised 72 species, 59 genera, and 31 families. Variations were detected regarding species and importance values obtained according to the topographic gradient. In the Canonical Correspondence Analysis, the coverage values of species showed significant correlation with the parameters of soil depth and slope.
\end{abstract}

Keywords: phytosociology, arboreal stratum, topographic gradient. 


\section{INTRODUÇÃO}

Na Argentina, a Província de Misiones encontra-se entre um dos pontos que abriga a maior biodiversidade do país. Nesta, segundo a classificação estabelecida pelo IBGE (2012), ocorrem as formações de Floresta Estacional Semidecidual, Floresta Ombrófila Mista e Estepe Estacional, sendo a primeira dessas formações a que cobre a maior parte de superfície provincial.

Visando à conservação dos recursos naturais e da diversidade cultural, o Estado, nacional e provincial, estabeleceu marcos legais para a criação de unidades de conservação no âmbito do território. Assim, entre as diversas categorias de proteção, existem atualmente 32 áreas de conservação (Di Bitetti et al., 2003).

Nesse contexto, os estudos florísticos e fitossociológicos proporcionam maior conhecimento sobre a diversidade de um local e descrições estruturais das comunidades vegetais, explicitam seu valor de conservação e geram melhor compreensão para as ações de manejo de um local, constituindo uma ferramenta relevante em unidades de conservação que contêm espécies de caráter endêmico ou de distribuição restrita.
O estudo de gradientes ambientais é um enfoque para a pesquisa de padrões espaciais da vegetação (Whittaker, 1967), em que a declividade tem demostrado influência dessa variável sobre a distribuição das espécies (Espírito-Santo et al., 2002; Botrel et al., 2002; Carvalho et al., 2005).

Nesse contexto, por meio de múltiplas pesquisas, tem-se revelado, ao longo dos anos, que a flora no Departamento de San Ignacio se distingue da restante da Província, com inúmeras espécies exclusivas e outras tantas endêmicas. Assim, o presente trabalho tem como objetivo conhecer a flora arbórea de uma unidade de conservação da Província de Misiones, por meio da compartimentação geomorfológica e subsequente estudo florístico e fitossociológico.

\section{MATERIAL E MÉTODO}

\section{1. Área de estudo}

O presente estudo foi desenvolvido em uma área de 40 hectares, dentro da Reserva Privada Osununú. Esta se encontra localizada no Departamento e Município de San Ignacio, $27^{\circ} 16^{\prime}$ S e $55^{\circ} 34^{\prime} \mathrm{O}$, nas margens do Rio Paraná, Província de Misiones, Argentina (Figura 1).

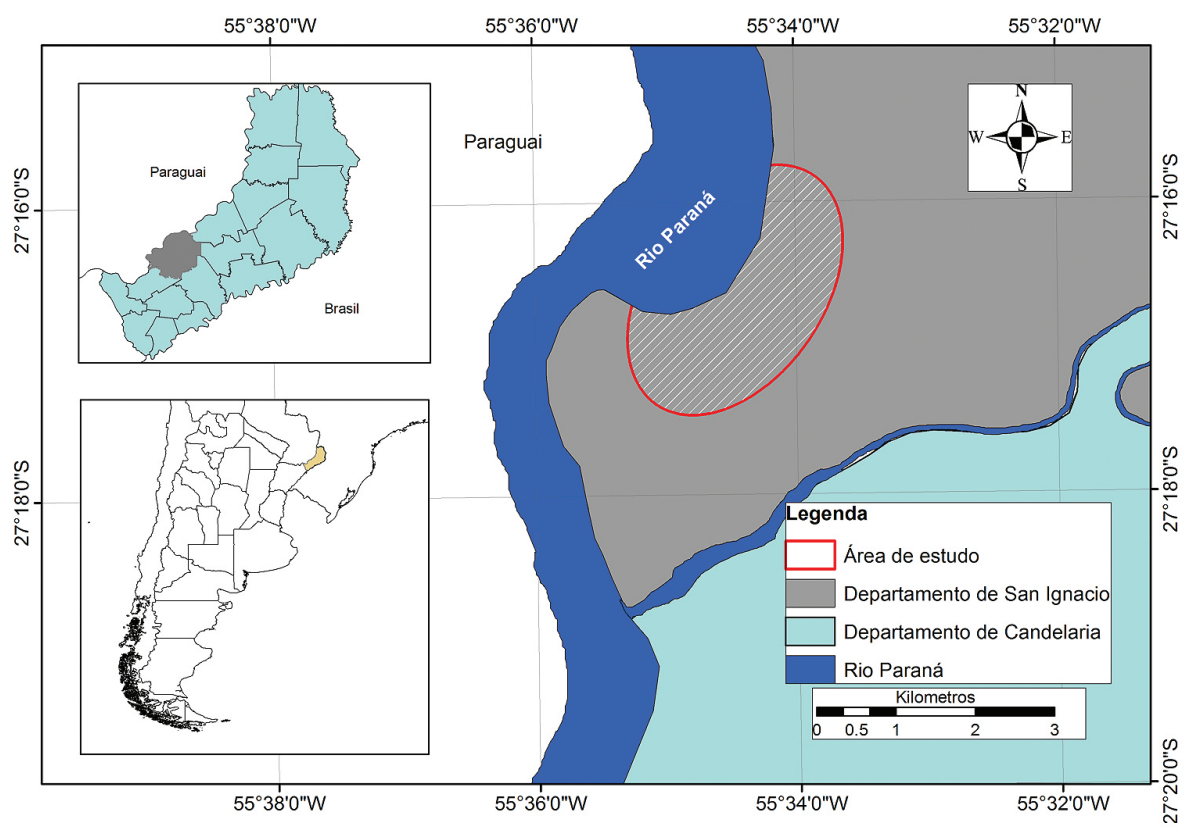

Figura 1. Localização geográfica da Reserva Privada Osununú, Departamento de San Ignacio, Província de Misiones, Argentina.

Figure 1. Geographical localization of the Osununú Private Reserve, San Ignacio department, Misiones province, Argentina. 
A reserva conta com uma superfície de 174 hectares, adjacente ao Parque Provincial Teyú Cuaré.

\subsection{Clima}

Segundo a classificação climática de Köppen, o clima da região é mesotérmico-úmido ( $\mathrm{Cfa}$ ). A temperatura registrada na localidade de Loreto, próxima a San Ignacio, é de $20,3{ }^{\circ} \mathrm{C}$ como média anual, $25,3{ }^{\circ} \mathrm{C}$ a média do mês mais quente e $14,9^{\circ} \mathrm{C}$ para o mês mais frio, com uma precipitação média anual de $1.700 \mathrm{~mm}$ (Martínez-Crovetto, 1963).

\subsection{Geomorfologia}

A área destaca-se no aspecto geológico, em que ocorrem afloramentos do Arenito Misiones, que faz parte da Formação Botucatu. No contexto regional, esta é uma característica importante, já que a maior parte da superfície da Província de Misiones está coberta por basalto da Formação Serra Geral. O arenito encontra-se em afloramentos às margens do Rio Paraná, com formação de morros que podem alcançar até $120 \mathrm{~m}$ de altura, sendo, portanto, constituído de um relevo fortemente ondulado (CARTA, 1962-1963).

\subsection{Solo}

Os processos pedogenéticos, que tomaram como material parental essa rocha sedimentar, deram como resultado solos majoritariamente arenosos, da ordem dos NEOSSOLOS LITÓLICOS ou REGOLÍTICOS. Segundo SAGyP (1990), os solos apresentam-se excessivamente drenados, fortemente ácidos, pobres em nutrientes e susceptíveis à erosão, sendo de escassa significância geográfica e restringindo-se praticamente aos arredores da localidade de San Ignacio.

\subsection{Vegetação}

Considerada como Floresta Estacional Semidecidual, as particularidades geológicas e pedológicas imperantes nessa porção da Província permitiram que se perpetuasse uma vegetação com várias singularidades florísticas.

Assim, foram citadas cinco espécies endêmicas para o Parque Provincial Teyú Cuaré e imediações: Gaya kelleri Krapov. (Krapovickas, 2012a); Hyptis australis Epling (Epling, 1949); Mesosetum comatum Swallen (Filgueiras, 1990); Sida rhizomatosa Krapov. (Krapovickas, 2012b), e Vernonia teyucuarensis Cabrera (Cabrera,
1987), e 23 espécies exclusivas, ou seja, espécies que são comuns em outros países, mas que, na Argentina, têm uma distribuição restrita. Algumas destas são: Ilex affinis Gardner (Keller \& Giberti, 2011); Acosmium subelegans (Mohlenbr.) Yakovlev; Qualea cordata Spreng. (Biganzoli \& Romero, 2004), e Calophyllum brasiliense Cambess. (Rodríguez et al., 2009).

\subsection{Método de amostragem}

Em uma primeira etapa, foi realizada a compartimentação por meio de fotografias aéreas e, para uma melhor visualização do gradiente topográfico, a partir de curvas de nível, foi gerado um DTM (Digital Terrain Model), com definição de $5 \mathrm{~m}$. As maiores declividades estão representadas por cores mais escuras na Figura 2. Assim, foram definidos quatro compartimentos que acompanharam o gradiente topográfico, desde as partes inferiores até as superiores. Em cada compartimento, foi descrito um perfil e tomadas amostras de solo para posterior identificação, segundo o Sistema Brasileiro de Classificação de Solos (EMBRAPA, 2013).

O Compartimento I correspondeu às áreas situadas na parte inferior do vale e às margens do Rio Paraná, com solos profundos, com abundante umidade conferida pela drenagem natural da encosta, assim como pela superficialidade do lençol freático. Tais solos têm sua origem na acumulação de sedimentos aparentemente de origem colúvio-aluvionar, ou seja, sedimentos transportados das partes superiores da paisagem e também da deposição pelo extravasamento do rio, sendo classificados como NEOSSOLO QUARTZARÊNICO Hidromórfico neofluvissólico.

O Compartimento II ficou definido pelo sopé das encostas, com declividades menores que $45 \%$. O perfil analisado foi classificado como um NEOSSOLO REGOLÍTICO Distrófico espessarênico. Os solos apresentaram profundidades entre 30 e $100 \mathrm{~cm}$, com média de $45 \mathrm{~cm}$. Este compartimento, ao estar precedido por encostas íngremes, atua como área deposicional de sedimentos coluviais.

O Compartimento III correspondeu àquelas zonas de encostas mais íngremes, com declividades superiores a $45 \%$, conformando-se, assim, áreas de deflúvio e erosionais. Os solos apresentaram-se rasos e geralmente pedregosos, com contato lítico a uma profundidade média de $25 \mathrm{~cm}$. Foram classificados como NEOSSOLO LITÓLICO Distrófico típico. 


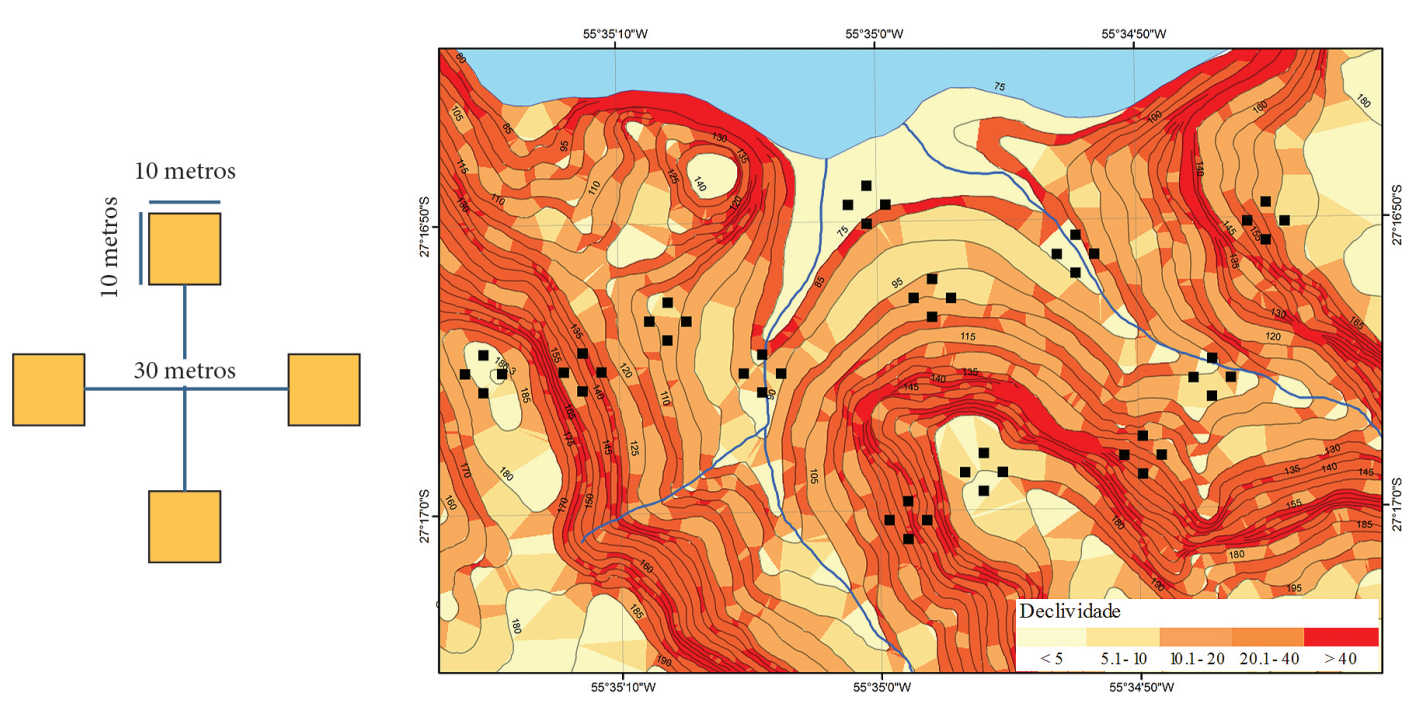

Figura 2. Estrutura, dimensões e distribuição dos conglomerados, e declividades representadas pelo gradiente de cores, na Reserva Privada Osununú.

Figure 2. Structure, dimensions and distribution of cluster, and slopes represented by gradient colors, in the Osununú Private Reserve.

O Compartimento IV caracteriza-se pela condição de topo, com solos geralmente pouco profundos, inferiores a $25 \mathrm{~cm}$, com pedregosidade no perfil e, em algumas unidades amostrais, com ocorrência de afloramento rochoso, constituindo-se NEOSSOLOS REGOLÍTICOS e NEOSSOLOS LITÓLICOS.

Foi utilizado o processo de amostragem em conglomerados, composta por quatro subunidades (parcelas) de $10 \times 10 \mathrm{~m}$, distanciadas $30 \mathrm{~m}$ entre si (Figura 2). Dentro de cada compartimento, foram instalados três conglomerados de maneira aleatória, totalizando, assim, 12 unidades amostrais por compartimento e 47 unidades para toda a área de estudo. A intensidade de amostragem utilizada foi 1,1\% em relação à área total da unidade de conservação.

Em cada parcela, foram mensurados todos os indivíduos arbóreos iguais ou maiores que $10 \mathrm{~cm}$ de perímetro à altura do peito (PAP), além de medidas as variáveis ambientais de profundidade do solo, por meio de um trado, e de declividade, com um hipsômetro digital.

\subsection{Processamento de dados}

Para cada indivíduo, foi identificado seu táxon em nível de espécie e atualizado seu nome e dos autores com base no Missouri Botanical Garden (http://www. tropicos.org).
Com o software EstimateS 9.1.0 (Colwell, 2013), foi confeccionada curva de rarefação, baseando-se nas unidades amostrais, e avaliada a exaustão amostral por meio dos estimadores de riqueza não paramétricos de Chao 2 e ICE (Incidence Coverage-based Estimator).

Por meio do software Florexel (Arce et al., 2002), foram avaliados os parâmetros fitossociológicos, tanto absolutos como relativos, de densidade, dominância e frequência, assim como o valor de importância.

Para avaliar a diversidade alfa das comunidades, foram utilizados os seguintes índices: índice de Shannon $\left(\mathrm{H}^{\prime}\right)$, índice de Simpson (D) e índice de Berger-Parque (d). Foi empregado o teste estatístico de randomização Solow (1993) para a comparação dos índices entre os diferentes compartimentos.

Foi feita uma Análise de Correspondência Canônica (CCA) (Ter Braak, 1986), já que esta é uma das técnicas de análise de gradiente indicadas, quando o objetivo é obter uma relação entre variáveis ambientais e a abundância de espécies (Kent, 2012).

A primeira matriz foi montada de maneira que cada variável $X_{j}$ representou uma espécie, considerando-se somente aquelas com mais de dez indivíduos (30 espécies), sendo que cada valor $X_{i j}$ esteve representado pelo valor de cobertura de cada espécie para cada parcela $X_{i}$. Esta variável foi empregada por resumir dois parâmetros de uma espécie (sua densidade e sua dominância) em um 
mesmo número. A segunda matriz foi a das variáveis ambientais $\left(X_{j}\right)$, declividade e profundidade do solo, em que cada um de seus valores $\left(X_{i j}\right)$ correspondeu a uma unidade amostral $X_{i}$.

Foi efetuado o teste de permutação de Monte Carlo, com 999 aleatorizações, para a verificação da significância das correlações entre os valores de cobertura das espécies e as variáveis ambientais.

\section{RESULTADOS E DISCUSSÃO}

Baseado na Figura 3, pode-se apreciar uma tendência à estabilização da curva de rarefação, mas sem atingir ainda uma assíntota, já que os valores dos estimados não paramétricos e os observados não são os mesmos. Segundo o valor de Chao 2, estima-se que na floresta existiria um total de 81 espécies; entretanto, para o ICE, esse valor ascende para 88 . Mesmo assim, as espécies registradas representam $88 \%$ e $81 \%$ do total de espécies estimadas.

Dessa maneira, foi mensurado um total de 1.295 indivíduos, identificadas 72 espécies, distribuídas em 59 gêneros e 31 famílias (Tabela 1). As famílias que apresentaram maior número de espécies foram Fabaceae (dez espécies), Myrtaceae (nove) e Meliaceae (cinco), enquanto que as famílias mais abundantes foram Myrtaceae, Clusiaceae, Primulaceae e Vochysiaceae.

A flora da área estudada apresentou vários componentes considerados por Spichiger et al.
(1995) típicos das florestas da região do Alto Paraná no Paraguai, assim como do Distrito dos Louros, segundo Martínez-Crovetto (1963). São estes: Cedrela fissilis, Chrysophyllum gonocarpum, Chrysophyllum marginatum, Cordia trichotoma, Guarea macrophylla, Helietta apiculata, Matayba elaeagnoides, Nectandra megapotamica, Sorocea bonplandii, Trichilia elegans e Trichilia catigua, entre outros. No entanto, foram outras espécies que figuraram como as mais comuns na floresta estudada, as quais, em ordem decrescente de abundância, seriam: Rheedia brasiliensis, Eugenia psidiiflora, Myrsine balansae, Qualea cordata e Paramyrciaria delicatula.

Concernente aos resultados resumidos na Tabela 2 , no compartimento II, foram registradas 47 espécies; tal compartimento apresentou o maior valor para os índices de Shannon, Simpson e de Berger-Parquer, sendo todos estes significativos em relação aos demais compartimentos, à exceção do índice de BergerParker, quando comparado com o compartimento I. O compartimento I foi o segundo em termos de riqueza, assim como em valores de diversidade para os três índices supracitados, e apresentou diferença significativa para todos os demais compartimentos, exceto o IV. Este último foi o terceiro em termos de valores de diversidade e diferiu estatisticamente dos compartimentos II e III. O compartimento III apresentou 28 espécies, com uma diminuição considerável para todos os índices, exibindo assim diferença significativa em relação a todos os demais.

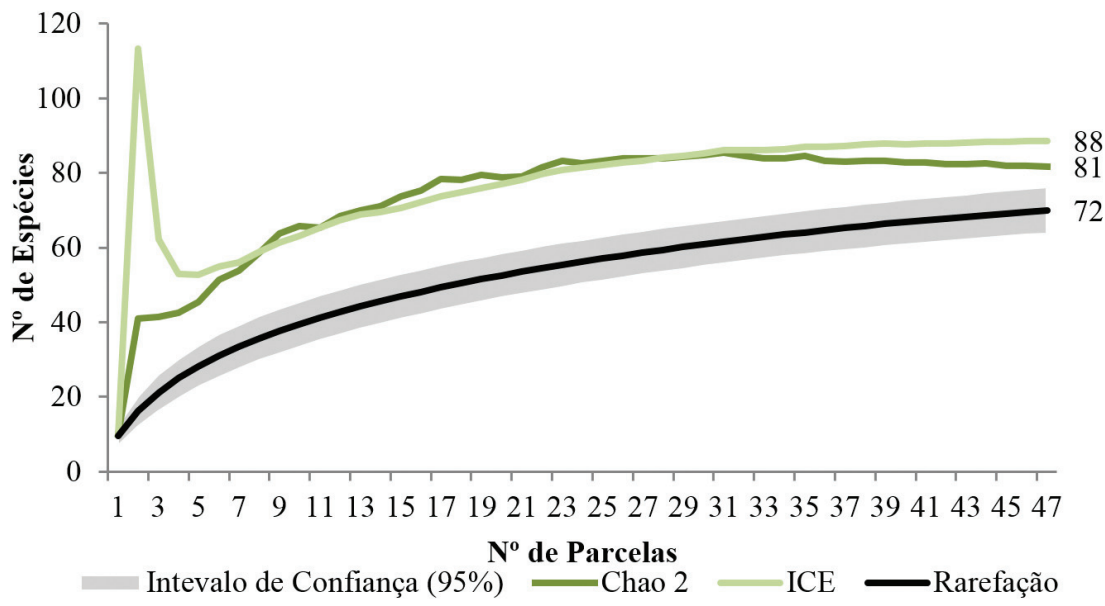

Figura 3. Curvas de rarefação, intervalo de confiança (95\%), estimadores ICE e CHAO 2, baseados nas amostras na Reserva Privada Osununú.

Figure 3. Curves of rarefaction, confidece interval (95\%), estimators of ICE and CHAO 2, based on samples in Osununú Private Reserve. 
Tabela 1. Famílias e espécies amostradas em cada compartimento, na Reserva Privada Osununú.

Table 1. Families and species sampled in each compartments, in Osununú Private Reserve.

\begin{tabular}{|c|c|c|c|c|c|}
\hline \multirow{2}{*}{ FAMÍLIA } & \multirow{2}{*}{ Espécie } & \multicolumn{4}{|c|}{ Compartimento } \\
\hline & & I & II & III & IV \\
\hline ANNONACEAE & Xylopia brasiliensis Spreng. & - & - & - & \\
\hline APOCYNACEAE & Tabernaemontana catharinensis A.DC. & $\bullet$ & & & $\bullet$ \\
\hline \multirow[t]{2}{*}{ AQUIFOLIACEAE } & Ilex affinis Gardner & $\bullet$ & & & \\
\hline & Ilex brevicuspis Reissek & $\bullet$ & $\bullet$ & $\bullet$ & $\bullet$ \\
\hline ARECACEAE & Syagrus romanzoffiana (Cham.) Glassman & $\bullet$ & $\bullet$ & $\bullet$ & $\bullet$ \\
\hline \multirow[t]{2}{*}{ BIGNONIACEAE } & Handroanthus heptaphyllus (Vell.) Mattos & - & & - & \\
\hline & Handroanthus pulcherrimus (Sandwith) Mattos & & & & $\bullet$ \\
\hline \multirow[t]{3}{*}{ BORAGINACEAE } & Cordia americana (L.) Gottschling \& J.S.Mill. & & $\bullet$ & & $\bullet$ \\
\hline & Cordia ecalyculata Vell. & & $\bullet$ & & \\
\hline & Cordia trichotoma (Vell.) Arráb. ex Steud. & $\bullet$ & $\bullet$ & & \\
\hline \multirow[t]{2}{*}{ CACTACEAE } & Brasiliopuntia schulzii (A. Cast. \& Lelong) Backeb. & & $\bullet$ & & \\
\hline & Cereus stenogonus K.Schum. & • & $\bullet$ & - & - \\
\hline CALOPHYLLACEAE & Calophyllum brasiliense Cambess. & $\bullet$ & $\bullet$ & $\bullet$ & \\
\hline CARICACEAE & Jacaratia spinosa (Aubl.) A.DC. & & $\bullet$ & & \\
\hline CLUSIACEAE & Rheedia brasiliensis (Mart.) Planch. \& Triana & $\bullet$ & $\bullet$ & $\bullet$ & $\bullet$ \\
\hline ERYTHROXYLACEAE & Erythroxylum deciduum A.St.-Hil. & & & $\bullet$ & \\
\hline \multirow{4}{*}{ EUPHORBIACEAE } & Actinostemon concolor (Spreng.) Müll.Arg. & & $\bullet$ & & $\bullet$ \\
\hline & Alchornea glandulosa Poepp. \& Endl. & $\bullet$ & $\bullet$ & $\bullet$ & $\bullet$ \\
\hline & Alchornea triplinervia (Spreng.) Müll.Arg. & $\bullet$ & $\bullet$ & $\bullet$ & $\bullet$ \\
\hline & Sebastiania brasiliensis Spreng. & & & & - \\
\hline \multirow[t]{10}{*}{ FABACEAE } & Acosmium subelegans (Mohlenbr.) Yakovlev & & $\bullet$ & & - \\
\hline & Apuleia leiocarpa (Vogel) J.F. Macbr. & & & & $\bullet$ \\
\hline & Calliandra foliolosa Benth. & & $\bullet$ & & \\
\hline & Inga vera subsp. affinis (DC.) T.D.Penn. & $\bullet$ & $\bullet$ & & \\
\hline & Lonchocarpus campestris Mart. ex Benth. & & & & $\bullet$ \\
\hline & Machaerium paraguariense Hassl. & & & & \\
\hline & Machaerium stipitatum Vogel & - & & & \\
\hline & Machaerium aculeatum Raddi & $\bullet$ & & & $\bullet$ \\
\hline & Myrocarpus frondosus Allemão & & & & $\bullet$ \\
\hline & Parapiptadenia rigida (Benth.) Brenan & & $\bullet$ & & \\
\hline \multirow[t]{4}{*}{ LAURACEAE } & Nectandra megapotamica (Spreng.) Mez & $\bullet$ & $\bullet$ & & \\
\hline & Ocotea diospyrifolia (Meisn.) Mez & & $\bullet$ & & \\
\hline & Ocotea lancifolia (Schott) Mez & $\bullet$ & $\bullet$ & $\bullet$ & \\
\hline & Ocotea puberula (Rich.) Nees & - & - & & \\
\hline MALVACEAE & Luehea divaricata Mart. \& Zucc. & & & & - \\
\hline MELASTOMATACEAE & Miconia pusilliflora (DC.) Naudin & $\bullet$ & & & \\
\hline \multirow[t]{5}{*}{ MELIACEAE } & Cabralea canjerana (Vell.) Mart. & $\bullet$ & $\bullet$ & $\bullet$ & \\
\hline & Cedrela fissilis Vell. & & $\bullet$ & & \\
\hline & Guarea macrophylla Vahl & $\bullet$ & $\bullet$ & $\bullet$ & $\bullet$ \\
\hline & Trichilia catigua A.Juss. & & $\bullet$ & & • \\
\hline & Trichilia elegans A.Juss. & & & & - \\
\hline \multirow[t]{3}{*}{ MORACEAE } & Ficus luschnathiana (Miq.) Miq. & $\bullet$ & $\bullet$ & & \\
\hline & Sorocea bonplandii (Baill.) W.C. Burger, & & & & \\
\hline & Lanj. \& Wess. Boer & & $\bullet$ & $\bullet$ & $\bullet$ \\
\hline \multirow[t]{4}{*}{ MYRTACEAE } & Calycorectes psidiiflorus (O. Berg) Sobral & & $\bullet$ & & • \\
\hline & Eugenia hyemalis Cambess. var. marginata & & & & \\
\hline & (O.Berg) D. Legrand & & & & $\bullet$ \\
\hline & Eugenia moraviana O. Berg & $\bullet$ & $\bullet$ & $\bullet$ & • \\
\hline
\end{tabular}


Tabela 1. Continuação...

Table 1. Continued...

\begin{tabular}{|c|c|c|c|c|c|}
\hline \multirow{2}{*}{ FAMÍLIA } & \multirow{2}{*}{ Espécie } & \multicolumn{4}{|c|}{ Compartimento } \\
\hline & & I & II & III & IV \\
\hline & Eugenia pyriformis Cambess. & $\bullet$ & $\bullet$ & $\bullet$ & $\bullet$ \\
\hline & Hexachlamys edulis (O. Berg) Kausel \& D. Legrand & $\bullet$ & & & $\bullet$ \\
\hline & Myrcia palustris DC. & $\bullet$ & $\bullet$ & $\bullet$ & $\bullet$ \\
\hline & Paramyrciaria delicatula (DC.) Kausel & & $\bullet$ & $\bullet$ & $\bullet$ \\
\hline & Plinia rivularis (Cambess.) Rotman & & $\bullet$ & - & - \\
\hline & Psidium guineense Sw. & - & & & \\
\hline PHYTOLACCACEAE & Seguiera aculeata Jacq. & & $\bullet$ & & \\
\hline POLYGONACEAE & Ruprechtia laxiflora Meisn. & & & & $\bullet$ \\
\hline PRIMULACEAE & Myrsine balansae (Mez) Otegui & • & $\bullet$ & - & - \\
\hline ROSACEAE & Prunus subcoriacea (Chodat \& Hassl.) Koehne & - & & & \\
\hline \multirow[t]{3}{*}{ RUBIACEAE } & Chomelia obtusa Cham. \& Schltdl. & & & & • \\
\hline & Coutarea hexandra (Jacq.) K.Schum. & & & $\bullet$ & $\bullet$ \\
\hline & Faramea cyanea Müll. Arg. & & $\bullet$ & & $\bullet$ \\
\hline \multirow[t]{2}{*}{ RUTACEAE } & Balfourodendron riedelianum (Engl.) Engl. & & $\bullet$ & & \\
\hline & Helietta apiculata Benth. & $\bullet$ & $\bullet$ & $\bullet$ & $\bullet$ \\
\hline SALICACEAE & Casearia sylvestris Sw. & - & $\bullet$ & - & $\bullet$ \\
\hline \multirow[t]{4}{*}{ SAPINDACEAE } & Allophylus edulis (A.St.-Hil. et al.) Hieron. ex Niederl. & & $\bullet$ & $\bullet$ & $\bullet$ \\
\hline & Cupania vernalis Cambess. & $\bullet$ & & & $\bullet$ \\
\hline & Diatenopteryx sorbifolia Radlk. & & $\bullet$ & • & $\bullet$ \\
\hline & Matayba elaeagnoides Radlk. & - & $\bullet$ & - & - \\
\hline \multirow{4}{*}{ SAPOTACEAE } & Chrysophyllum gonocarpum & \multirow{3}{*}{ • } & \multirow{3}{*}{ • } & \multirow{3}{*}{$\bullet$} & \multirow{3}{*}{$\bullet$} \\
\hline & (Mart. \& Eichler ex Miq.) Engl. & & & & \\
\hline & Chrysophyllum marginatum (Hook. \& Arn.) Radlk. & & & & \\
\hline & Pouteria gardneriana (A. DC.) Radlk. & $\bullet$ & $\bullet$ & & $\bullet$ \\
\hline SIMAROUBACEAE & Picrasma crenata (Vell.) Engl. & $\bullet$ & & & \\
\hline URTICACEAE & Cecropia pachystachya Trécul & - & & & \\
\hline VOCHYSIACEAE & Qualea cordata (Mart.) Spreng. & $\bullet$ & $\bullet$ & $\bullet$ & $\bullet$ \\
\hline
\end{tabular}

Tabela 2. Número de indivíduos (NI), Riqueza (S), Índice de Shannon ( $\left.\mathrm{H}^{\prime}\right)$, Complemento do Índice de Simpson (1-D) e Recíproca do Índice de Berger-Parquer (1/d) por compartimento na Reserva Privada Osununú.

Table 2. Number of individuals (NI), Number of species (S), Shannon's Index $\left(\mathrm{H}^{\prime}\right)$, Complement Simpson's Index (1-D) and Reciprocal Berger-Parquer's Index (1/d), values in the different compartments concerning in the Osununú Private Reserve.

\begin{tabular}{cccccc|} 
Compartimento & NI & S & H' $^{\prime}$ & 1-D & 1/d \\
\hline I & 293 & 39 & $3,01 \mathrm{~b}$ & $13,19 \mathrm{ab}$ & $4,78 \mathrm{~b}$ \\
\hline II & 339 & 47 & $3,20 \mathrm{a}$ & $17,14 \mathrm{a}$ & $7,91 \mathrm{a}$ \\
\hline III & 327 & 28 & $2,44 \mathrm{c}$ & $6,16 \mathrm{c}$ & $2,76 \mathrm{c}$ \\
\hline IV & 336 & 42 & $2,90 \mathrm{~b}$ & $11,45 \mathrm{~b}$ & $4,72 \mathrm{~b}$ \\
\hline Total & 1295 & 72 & & & \\
\hline
\end{tabular}

Valores seguidos da mesma letra no sentido vertical não diferem estatistícamente entre si no nível de 95\% de probabilidade. Values followed by the same letter in the vertical direction do not differ statistically at $95 \%$ probability.

A maior diversidade do segundo compartimento possivelmente pode derivar das condições pedológicas mais propícias, por se tratar de um compartimento com solos mais profundos que os situados em vertentes íngremes e em topos, e também sem influência do lençol freático, como ocorre nas planícies próximas ao rio.

Resultados semelhantes foram obtidos por Souza et al. (2013) num gradiente topográfico em Floresta Estacional 
Semidecidual, em que as áreas planas e com menor erosão mostraram índices maiores de diversidade $\mathrm{e}$ um maior número de espécies raras.

$\mathrm{Na}$ transição entre os compartimentos II e III, é notória a diminuição da riqueza, assim como da diversidade (Figura 4 e Tabela 3). Esse declínio de diversidade nas áreas de encostas foi semelhante aos resultados obtidos por Botrel et al. (2002) e Souza et al. (2013), em outras florestas estacionais. Ao contrário da situação dos compartimentos I e II, as condições

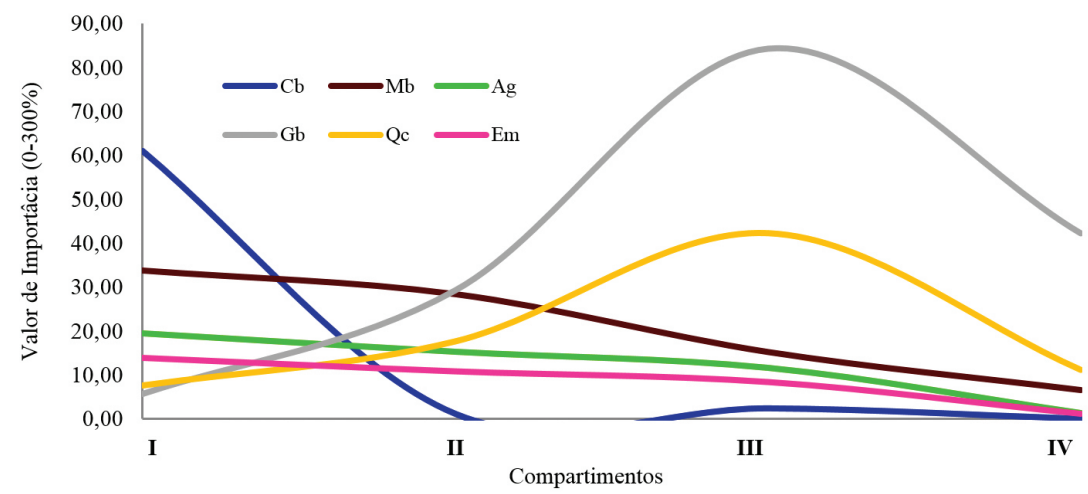

Figura 4. Distribuição do Valor de Importância das espécies Calophyllum brasiliense (Cb); Myrsine balansae (Mb); Alchornea glandulosa (Ag); Eugenia moraviana (Em); Rheedia brasiliensis (Gb) e Qualea cordata (Qc) nos quatro compartimentos na Reserva Privada Osununú.

Figure 4. Distribution of the species importance value for Calophyllum brasiliense (Cb); Myrsine balansae (Mb); Alchornea glandulosa (Ag); Eugenia moraviana (Em); Rheedia brasiliensis (Gb) e Qualea cordata (Qc) in the four compartments, in the Osununú Private Reserve.

Tabela 3. Densidades, dominâncias e frequências absolutas e relativas das cinco espécies com maior valor de importância para os quatro compartimentos na Reserva Privada Osununú.

Table 3. Density, dominance and frequency absolute and relative of the five species with highest important values, for the four compartments, in the Osununú Private Reserve.

\begin{tabular}{|c|c|c|c|c|c|c|c|c|}
\hline Compartimento & Espécie & DA & DR & DoA & DoR & FA & FR & VI \\
\hline \multirow{5}{*}{$\mathrm{I}$} & Calophyllum brasiliense & 183,33 & 7,51 & 19,06 & 48,02 & 58,33 & 5,34 & 60,88 \\
\hline & Myrsine balansae & 491,67 & 20,14 & 2,68 & 6,74 & 75 & 6,87 & 33,75 \\
\hline & Alchornea glandulosa & 141,67 & 5,8 & 2,25 & 5,68 & 66,67 & 6,11 & 17,58 \\
\hline & Xylopia brasiliensis & 216,67 & 8,87 & 0,93 & 2,33 & 58,33 & 5,34 & 16,55 \\
\hline & Guarea macrophylla & 150 & 6,14 & 0,43 & 1,09 & 75 & 6,87 & 14,1 \\
\hline \multirow{5}{*}{ II } & Rheedia brasiliensis & 341,67 & 12,09 & 1,93 & 8,41 & 91,67 & 8,66 & 29,16 \\
\hline & Myrsine balansae & 333,33 & 11,8 & 2,56 & 11,15 & 50 & 4,72 & 27,67 \\
\hline & Qualea cordata & 266,67 & 9,44 & 0,85 & 3,71 & 41,67 & 3,94 & 17,09 \\
\hline & Alchornea glandulosa & 58,33 & 2,06 & 2,5 & 10,85 & 25 & 2,36 & 15,28 \\
\hline & Myrcia palustris & 183,33 & 6,49 & 0,55 & 2,38 & 33,33 & 3,15 & 12,02 \\
\hline \multirow{5}{*}{ III } & Rheedia brasiliensis & 1018,18 & 34,25 & 6,78 & 36 & 100 & 10 & 80,25 \\
\hline & Qualea cordata & 318,18 & 10,7 & 4,15 & 22,03 & 90,91 & 9,09 & 41,83 \\
\hline & Syagrus romanzoffiana & 109,09 & 3,67 & 1,49 & 7,92 & 72,73 & 7,27 & 18,86 \\
\hline & Myrsine balansae & 136,36 & 4,59 & 0,81 & 4,31 & 72,73 & 7,27 & 16,17 \\
\hline & Paramyrciaria delicatula & 236,36 & 7,95 & 0,81 & 4,3 & 36,36 & 3,64 & 15,89 \\
\hline \multirow{5}{*}{ IV } & Rheedia brasiliensis & 575 & 20,54 & 2,36 & 12,98 & 83,33 & 8,7 & 42,21 \\
\hline & Eugenia psidiiflora & 341,67 & 12,2 & 1,48 & 8,15 & 41,67 & 4,35 & 24,7 \\
\hline & Helietta apiculata & 225 & 8,04 & 1,08 & 5,95 & 75 & 7,83 & 21,82 \\
\hline & Plinia rivularis & 216,67 & 7,74 & 1,55 & 8,54 & 50 & 5,22 & 21,5 \\
\hline & Paramyrciaria delicatula & 166,67 & 5,95 & 1,23 & 6,77 & 41,67 & 4,35 & 17,07 \\
\hline
\end{tabular}

DA: densidade absoluta (Ind/ha); DR: densidade relativa (\%); DoA: dominância absoluta ( $\mathrm{m}^{2} / \mathrm{ha}$ ); DoR: dominância relativa (\%); FA: frequência absoluta (\%); FR: frequência relativa (\%); VI: valor de importância (\%0-300).

DA: absolute density (Ind/ha); DR: relative density (\%); DoA: absolute dominance ( $\left.\mathrm{m}^{2} / \mathrm{ha}\right)$; DoR: relative dominance (\%); FA: absolute frequency (\%); FR: relative frequency (\%); VI: importance value (\%0-300). 
mais rigorosas de declividade e de solos mais rasos parecem ser impedimentos para muitas espécies, razão que facilita a presença de táxons dominantes.

No compartimento I, os maiores valores de importância foram para Calophyllum brasiliense, Myrsine balansae e Alchornea glandulosa; já nas áreas com declividade média (compartimento II), destacou-se Rheedia brasiliensis, seguida por Myrsine balansae e Qualea cordata. Nas áreas mais íngremes (compartimento III), se destacou Rheedia brasiliensis - o que se justifica, evidentemente, pela elevada densidade e dominância - seguida de Qualea cordata e Syagrus romanzoffiana. No caso do compartimento IV, o maior valor de importância foi para Rheedia brasiliensis, Eugenia psidiiflora e Helietta piculata (Tabela 3).

O elevado valor de importância apresentado por Calophyllum brasiliense se deveu à presença de indivíduos de grande porte, resultando em altos valores de dominância. Há estudos em que a espécie foi uma das de maior valor de importância em ambientes de restinga não inundável (Guedes et al., 2006) ou em áreas ripárias de Floresta Estacional Semidecidual (Toniato et al., 1998; Costa Filho et al., 2006; OliveiraFilho et al., 1994) ou mesmo em caxetais (Galvão et al., 2002), em razão, justamente, da sua dominância.

A ocorrência natural dessa espécie em solos com drenagem deficiente, ou então periodicamente inundáveis, fica evidenciada pela diminuição do valor de importância, o qual decai de forma notável nos compartimentos de encosta (Figura 4).

Deve-se salientar que, no compartimento I, Calophyllum brasiliense se mostrou fortemente associada a dois córregos, ambientes mais úmidos deste setor, onde era comum um sub-bosque dominado por Blechnum brasiliense Desv. A ocorrência agregada dessa espécie foi observada por Rodríguez et al. (2009) em San Ignacio e por Bertoni et al. (1994) e Lopez (1979), no Paraguai.

Myrsine balansae (Figura 4) apresenta uma tolerância menor a ambientes excessivamente úmidos, pelo que teve maior expressividade nos ambientes afastados dos corpos d'água do primeiro compartimento. $\mathrm{O}$ valor de importância dessa espécie, junto a Alchornea gladulosa e Eugenia moraviana, expressou um declínio no sentido contrário ao gradiente topográfico (Figura 4). Esse comportamento similar das três espécies pode estar demostrando a exigência destas por solos mais profundos, sem impedimento mecânico e mais raramente submetidos a um déficit hídrico. Alchornea glandulosa, segundo Smith et al. (1988), é uma espécie que ocorre principalmente ao longo dos rios ou nas planícies aluviais, em orlas das florestas; essa espécie é considerada por Spichiger et al. (1992) como uma espécie heliófila, abundante nas áreas ripárias do Rio Paraná.

É notória a expressividade de Rheedia brasiliensis e Qualea cordata nas áreas com as maiores declividades (Figura 4). No caso da primeira espécie, esse resultado apresenta-se discrepante quando comparado a outros autores, que a caracterizam como espécie seletiva higrófila (Lorenzi, 2009) e que frequenta áreas úmidas (Spichiger et al., 1992; Lopez, 1979).

Qualea cordata é uma espécie comum em cerrados e geralmente encontra-se em solos rasos e ambientes relativamente secos (Yamamoto, 2009; Negrelle, 2011); contudo, apresenta certa plasticidade, o que lhe permite se estabelecer em diferentes condições ambientais (Figura 4). Segundo Spichiger et al. (1995), essa espécie, junto com Vochysia tucanorum, são as duas Vochysiaceae mais frequentes em áreas de savanas, florestas abertas e de galerias, nas regiões de cerrado, no Paraguai oriental.

Gomidesia palustres, aparentemente, está bem adaptada a solos arenosos, já que, segundo Legrand \& Klein (1967), ocorre nas restingas litorâneas e nos terrenos arenosos.

Segundo Spichiger et al. (1995), Plinia rivularis é uma espécie que ocorre com frequência em morros com solos rasos ou com afloramento, embora também seja mencionada como uma das espécies mais importantes em florestas ribeirinhas e também sobre solos mal drenados, em florestas no Paraguai oriental (Spichiger et al., 1992; Jiménez et al., 2002).

Eugenia psidiiflora foi abundante nas parcelas de solos mais rasos e com afloramento rochoso do compartimento I. A mesma foi caracterizada como de ocorrência pouco frequente, que habita florestas ribeirinhas e afloramentos rochosos, e barrancos dos rios (Legrand \& Klein, 1972; Rotman, 1982).

No diagrama de ordenação resultante da CCA (Figura 5), denota-se claramente a agregação da maioria das unidades amostrais, conforme os compartimentos, para os dois primeiros eixos. 


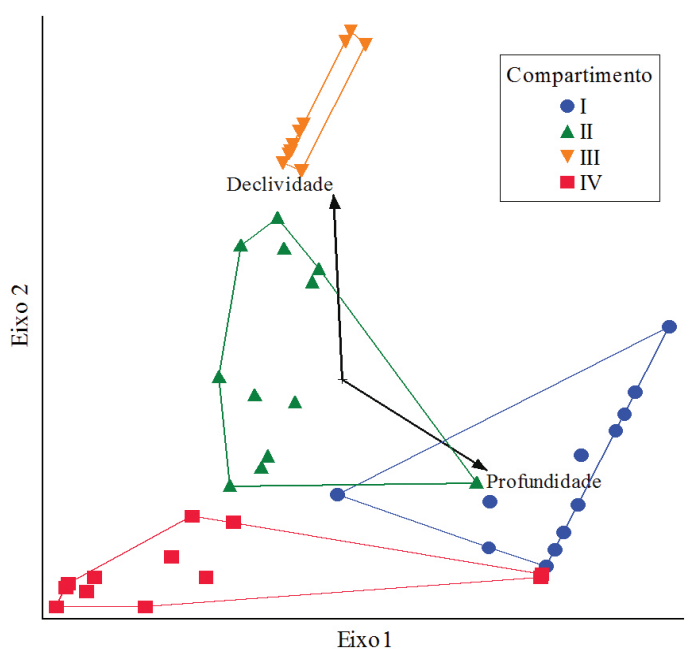

Figura 5. Diagrama de ordenação para os dois primeiros eixos da análise de correspondência canônica (CCA).

Figure 5. Ordination diagram for the first two axes of the canonical correspondence analysis (CCA).

Tabela 4. Resumo da análise de correspondência canônica (CCA).

Table 4. Summary of the canonical correspondence analysis (CCA).

\begin{tabular}{lcc} 
& Eixo 1 & Eixo 2 \\
\hline Autovalor & 0,45 & 0,23 \\
\hline Variância acumulada (\%). & 8,8 & 13,5 \\
\hline Correlação de Pearson & 0,87 & 0,69 \\
Profundidade & 0,87 & $-0,49$ \\
Declividade & $-0,05$ & 0,99 \\
Teste de Monte Carlo & 0,001 & \\
\hline
\end{tabular}

Segundo os dados da Tabela 4, o primeiro eixo está fortemente correlacionado com a profundidade do solo, enquanto que o segundo eixo tem uma maior correlação com a variável declividade. Considerando-se os dois eixos de ordenação, só foi possível captar $13,50 \%$ da variabilidade dos dados, mas mesmo assim o teste de permutação de Monte Carlo resultou numa correlação significativa entre as variáveis ambientais e o valor de cobertura.

A variabilidade captada nos eixos de ordenação foi igual ou menor, quando comparada a outras pesquisas realizadas, que utilizaram CCA (Botrel et al., 2002; Carvalho et al., 2005; Espírito-Santo et al., 2002). Mesmo assim, os resultados foram satisfatórios, considerando-se que, na presente análise, só foram incluídas duas variáveis, enquanto que, nos demais estudos, foram incluídos fatores químicos e texturais do solo.
Como foi demostrado por Botrel et al. (2002) e Carvalho et al. (2005), estudando o gradiente de solos e topográficos em Floresta Estacional Semidecidual, os fatores que mais influenciaram na distribuição das espécies foram o regime de água e a fertilidade química do solo.

No caso da área estudada, a água, embora não avaliada, também poderia estar influenciando a distribuição das espécies. A atuação conjunta entre a forte declividade e a predominância de solos arenosos favorece a drenagem, o que propicia um gradiente hídrico marcado, em que as encostas escarpadas e os cumes apresentam-se com ambientes muito mais secos, contrapondo-se às partes inferiores do vale.

\section{CONCLUSÕES}

Ao longo do gradiente topográfico, foi possível detectar variações a respeito da diversidade de espécies, assim como das variáveis fitossociológicas.

No compartimento I, o maior valor de importância foi para Calophyllum brasiliense, seguido por Myrsine balansae e Alchornea glandulosa. Nas áreas com declividade média, as espécies mais importantes foram Rheedia brasiliensis, Myrsine balansae e Qualea cordata, enquanto que, nos locais mais íngremes, se destacaram Rheedia brasiliensis e Qualea cordata. Já nas áreas altas da paisagem, sobressaíram Rheedia brasiliensis, Eugenia psidiiflora e Helietta apiculata.

Segundo a análise CCA, os valores de cobertura das espécies mostraram ter uma correlação significativa com os parâmetros profundidade de solo e declividade. A baixa variabilidade explicada nos eixos de ordenação indica a existência de uma complexidade maior de fatores que atuam como determinantes da estruturação da flora, em cada compartimento.

O alto valor de importância das espécies exclusivas e pouco frequentes, como Calophyllum brasiliense, Qualea cordata e Xylopia brasiliensis, denota a singularidade florística e estrutural da reserva, tornando-a singular em nível provincial.

\section{AGRADECIMENTOS}

À Facultad de Ciencias Forestales, da Universidad Nacional de Misiones, pelo apoio econômico. Ao Ministerio de Ecología y Recursos Naturales Renovables 
y Turismo de la Provincia de Misiones, pela autorização para o acesso às unidades de conservação. À Fundación Temaikén, pela autorização concedida para realizar esta pesquisa na sua reserva. Ao Ministerio del Agro y la Producción, pelos materiais fotográficos disponibilizados. À Licenciada María Paula Bertolini, pelo apoio logístico e as inúmeras ajudas oferecidas. À Nancy Paredes, pelo auxílio no campo, assim como aos guarda-parques Renzo Ramirez, Oscar Flores e Raúl Flores. Ao Prof. Sylvio Pellico Netto (UFPR), pelas sugestões e revisão do trabalho.

\section{STATUS DA SUBMISSÃO}

Recebido: 10 abr., 2013

Aceito: 25 ago., 2014

\section{AUTOR(ES) PARA CORRESPONDÊNCIA}

\section{Santiago José Elías Velazco}

Departamento de Ciências Florestais, Universidade Federal do Paraná - UFPR, CEP 80210-170, Curitiba, PR, Brasil e-mail: sjevelazco@gmail.com

\section{REFERENNCIAS}

Arce JE, Koehler A, Jaster CB, Sanquetta CR. Florexel: funções florestais desenvolvidas para o Microsoft Excel@). Curitiba: Centro de Ciências Florestais e da Madeira CCFM, UFPR; 2002.

Bertoni S, Dure R, Florentin T, Pin A, Pinazzo J, Quintana M, et al. Flora Amenazada del Paraguay. Dirección de Parques Nacionales y Vida Silvestre; 1994.

Biganzoli F, Romero MEM. Inventario florístico del Parque Provincial Teyú Cuaré y Alrededores (Misiones, Argentina). Darwiniana 2004; 42(1-4): 1-24.

Botrel RT, Oliveira Filho AT, Rodrigues LA, Curi N. Influência do solo e topografia sobre as variações da composição florística e estrutura da comunidade arbóreo-arbustiva de uma floresta estacional semidecidual em Ingaí, MG. Brazilian Journal of Botany 2002; 25(2): 195-213.

Cabrera AL. Una nueva especie de Vernonia (Compositae) de Misiones, república Argentina. Darwiniana 1987; 28(1-4): 187-189.

Carvalho DA, Oliveira Filho AT, Vilela EA, Curi N, Van Den Berg E, Fontes MAL et al. Distribuição de espécies arbóreo-arbustivas ao longo de um gradiente de solos e topografia em um trecho de floresta ripária do Rio São
Francisco em Três Marias, MG, Brasil. Brazilian Journal of Botany 2005; 25(2): 329-345.

Colwell RK. EstimateS: statistical estimation of species richness and shared species from samples. Version 9.1.0. USA: University Connecticut; 2013. Available from: http:// viceroy.eeb.uconn.edu/estimates/.

Compañía Argentina de Relevamientos Topográficos y Aerofotogramétricos - CARTA. Mapa Geológico de la Provincia de Misiones 1:50.000. Argentina; 1962-1963. p. 2757-24-4.

Costa Filho LV, Nanni MR, Campos JB. Floristic and phytosociological description of a riparian forest and the relationship with the edaphic environment in Caiuá Ecological Station - Paraná - Brazil. Brazilian Archives of Biology and Technology 2006; 49(5): 785-798. http:// dx.doi.org/10.1590/S1516-89132006000600013.

Di Bitetti MS, Placci G, Dietz LA. Una visión de biodiversidad para la ecorregión del Bosque Atlántico del Alto Paraná: diseño de un paisaje para la conservación de la biodiversidad y prioridades para las acciones de conservación. Washington: World Wild Life Foundation; 2003.

Empresa Brasileira de Pesquisa Agropecuária - EMBRAPA. Centro Nacional de Pesquisa de Solos. Sistema brasileiro de classificação de solos. 2. ed Rio de Janeiro; 2013.

Epling CC. Revisión del género Hyptis (Labiatae). Revista del Museo de la Plata: Sección Botánica 1949; 7: 153-497.

Espírito-Santo FB, Oliveira-Filho AT, Machado ELM, Souza JS, Fontes MAL, Sá JJG, et al. Variáveis ambientais e a distribuição de espécies arbóreas em um remanescente de Floresta Estacional Semidecídua Montana no campus da Universidade Federal de Lavras, MG. Acta Botanica Brasilica. 2002; 26(3): 331-356.

Filgueiras TS. Revisão de Mesosetum Steudel (Gramineae: Paniceae). Acta Amazonica 1990; 19: 47-114.

Galvão F, Roderjan CV, Yoshiko SK, Ziller SR. Composição florística e fitossociologica de caxetais do litoral do estado do Paraná - Brasil. Floresta 2002; 32(1): 17-39.

Guedes D, Barbosa LM, Martins SE. Composição florística e estrutura fitossociológica de dois fragmentos de floresta de restinga no Município de Bertioga, SP, Brasil. Acta Botanica Brasilica 2006; 20(2): 299-311.

Instituto Brasileiro de Geografia e Estatística - IBGE. Coordenação de Recursos Naturais e Estudos Ambientais. Manual técnico da vegetação brasileira. 2. ed. Rio de Janeiro; 2012. Manuais Técnicos em Geociências.

Jiménez B, Knapp S, Marín G, Peña-Chocarro M. Listado preliminar de plantas vasculares de la Reserva Natural del Bosque Mbaracayú, Paraguay. Rojasiana 2002; 5(2), 101-290.

Keller H, Giberti G. Primer registro para la flora Argentina de (Aquifoliaceae), Sustituto de la "Yerba Mate". Boletín Sociedad Argentina de Botánica 2011; 46(1-2): 187-194. 
Kent M. Vegetation description and data analysis: a practical approach. 2nd ed. Wiley-Blackwell; 2012.

Krapovickas A. Novedades en el género Gaya (Malvaceae). Bonplandia 2012a; 21(1): 71-76.

Krapovickas A. Novedades en las secciones Cordifoliae, Distichifolia, Malacroidea, Muticae y Nelavaga. Del género Sida (Malvaceae). Bonplandia 2012b; 21(1): 77-92.

Legrand CD, Klein RM. Mirtáceas. Itajaí: Herbário Barbosa Rodrigues; 1967. Flora Ilustrada Catarinense.

Legrand CD, Klein RM. Mirtáceas. Itajaí: Herbário Barbosa Rodrigues; 1972. Flora Ilustrada Catarinense.

Lopez JA. Arboles de la región oriental del Paraguay: nociones sobre dendrología. Asunción; 1979.

Lorenzi H. Árvores brasileiras: manual de identificação e cultivo de plantas arbóreas nativas do Brasil. Nova Odessa: Instituto Plantarum; 2009.

Martínez-Crovetto R. Esquema fitogeográfico de la Provincia de Misiones (República Argentina). Bonplandia 1963; 1(3).

Negrelle RRB. Qualea Aubl. from Paraná State, Brazil. Acta Scientiarum: Biological Sciences 2011; 33(3): 347-355.

Oliveira-Filho AT, Vilela EA, Carvalho DA, Gavilanes ML. Effects of soils and topography on the distribution of three species in a tropical riverine forest in touth-eastern Brazil. Journal of Tropical Ecology 1994; 10(04): 483-508. http://dx.doi.org/10.1017/S0266467400008178.

Rodríguez ME, Cardozo AE, Krauczuk ER, Fontana JL, Iriart D. Calophyllum brasiliense (CLUSIACEAE): Nuevo Registro Para la Flora de la Argentina. Boletín Sociedad Argentina de Botánica 2009; 44(3-4): 361-366.

Rotman AD. Los géneros Calycorectes, Hexachlamys, Myrciaria, Paramyrciaria, Plinia y Siphoneugena en la flora argentina. (Myrtaceae). Darwiniana 1982; 24(14): 157-185

SAGyP. INTA. Atlas de suelos de la República Argentina. Buenos Aires; 1990. Proyecto PNUD Arg-85/019. Tomo 1, 39 mapas.

Smith LB, Downs RJ, Klein RM. Euforbiáceas. Itajaí: Herbário Barbosa Rodrigues; 1988. Flora Ilustrada Catarinense.

Solow ARA. Simple test for change in community structure. Journal of Animal Ecology 1993; 62(1): 191-193.

Souza PB, Meira Neto JAA, Souza AL. Diversidade florística e estrutura fitossociológica de um gradiente topográfico em floresta estacional semidecidual submontana, MG. Cerne 2013; 19(3): 489-499. http://dx.doi.org/10.1590/ S0104-77602013000300017.

Spichiger R, Bertoni BS, Loizeau PA. The forest of Paraguayan Alto Parana. Candollea 1992; 47(2): 219-250.

Spichiger R, Palese R, Chautems A, Ramella L. Origin, affinities and diversity hot spots of the Paraugayan dendroflora. Cadollea 1995; 50(2): 515-537.

Ter Braak CJF. Canonical correspondence analysis: a new eigenvector technique for multivariate direct gradient analysis. Ecology 1986; 67(5): 1167-1179. http://dx.doi. org/10.2307/1938672.

Toniato MTZ, Leitão Filho HF, Rodrigues RR. Fitossociologia de um remanescente de floresta higrófila (mata de brejo) em Campinas, SP. Brazilian Journal of Botany 1998; 21(2): 1-17.

Whittaker RH. Gradient analysis of vegetation. Biological Reviews. 1967; 42(2): 207-264.

Yamamoto K. Flora de Grão-Mogol, Minas Gerais: Vochysiaceae. Boletim de Botânica da Universidade de São Paulo 2009; 27(1): 131-136. 